\title{
Multiple-phase spiral CT findings of pancreatic vasoactive intestinal peptide-secreting tumor: A case report
}

\author{
YING CHEN $^{1}$, DAN SHI $^{1}$, FEI DONG $^{1}$, SHU-GAO HAN $^{1}$, ZI-HUA QIAN $^{1}$, LI YANG $^{1}$, \\ YING WANG $^{1}$, RI-SHENG YU ${ }^{1}$, QING-HAI LI ${ }^{1}$ and YAN-BIAO FU ${ }^{2}$ \\ Departments of ${ }^{1}$ Radiology and ${ }^{2}$ Pathology, The Second Affiliated Hospital, \\ Zhejiang University School of Medicine, Hangzhou, Zhejiang 310009, P.R. China
}

Received July 30, 2014; Accepted July 28, 2015

DOI: $10.3892 / \mathrm{ol} .2015 .3615$

\begin{abstract}
The present study reports a case of pancreatic vasoactive intestinal peptide-secreting tumor (VIPoma), of $2.2 \mathrm{~cm}$ in diameter, arising from the region of the uncus of the pancreas with liver metastases in a 50-year-old patient, which demonstrated unusual multiple-phase spiral computed tomography (MPSCT) results. The pancreatic lesion was isodense compared with the pancreatic parenchyma. During the hepatic artery phase and portal venous phase, the mass was hypodense compared with the enhanced pancreas, with the mean $\mathrm{CT}$ attenuation ( $\mathrm{HU}$ ) values being $56 \mathrm{HU}$ and $66 \mathrm{HU}$, respectively. During the hepatic parenchymal phase, the mass became hyperdense with the mean CT attenuation values being $74 \mathrm{HU}$. The process of contrast-enhanced MPSCT demonstrated progressive strengthening. Pancreaticoduodenectomy and wedge resection of a number of the liver metastases was performed. There are only a limited number of studies reporting CT findings of pancreatic VIPoma and no MPSCT findings have been described previously.
\end{abstract}

\section{Introduction}

Vasoactive intestinal peptide-secreting tumors (VIPomas) are one of the least common types of pancreatic functioning islet cell tumors. The annual incidence of these tumors is estimated to $\sim 1$ per 10 million individuals in the general population (1). The definitive diagnosis of VIPoma is established by the laboratory evaluation of plasma VIP level (2). Imaging studies also serve important roles in determining size, location of islet cell tumors, optimal therapy, shortening operative time, and avoiding unnecessary resection of the pancreas. However, only a limited number of previous studies have reported computed

Correspondence to: Dr Ri-Sheng Yu, Department of Radiology, The Second Affiliated Hospital, Zhejiang University School of Medicine, 88 Jiefang Road, Hangzhou, Zhejiang 310009, P.R. China E-mail: cjr.yurisheng@vip.163.com

Key words: pancreas, computed tomography, vasoactive intestinal peptide-secreting tumor, liver, neoplasm tomography (CT) findings of pancreatic VIPoma (3-5). The radiological manifestations of multiple-phase spiral computed tomography (MPSCT) have not previously been described. The present case study reports a rare case of pancreatic VIPoma with liver metastases and focusses on the imaging features of the tumor, particularly the MPSCT findings, in a 50-year-old patient, and the current literature is reviewed. Written informed consent was obtained from the patient and the study was approved by the ethics committee of The Second Affiliated Hospital of Zhejiang University School of Medicine (Hangzhou, China).

\section{Case report}

A 50-year-old woman was admitted to The Second Affiliated Hospital of Zhejiang University School of Medicine on March 1, 2013 following a ten-month history of fatigue, weakness and diarrhea. The patient did not present with abdominal pains or fever. No positive findings, such as yellow sclera and skin, abdominal and rebound tenderness, or general superficial lymph node enlargement, were observed upon physical examination. Laboratory tests demonstrated marked hypokalemia (2.4 mmol/1, normal range: $3.5-5.5 \mathrm{mmol} / \mathrm{l})$ and hyperglycemia $(7.7 \mathrm{mmol} / 1$, normal range: 5.0-5.6 $\mathrm{mmol} / \mathrm{l})$. The other hormone levels of the patient included normal glucagon, gastrin, and insulin levels. In addition, the expression levels of the tumor markers CEA and CA19-9 were normal. Transabdominal ultrasound detected a $2.0 \mathrm{~cm}$ round lesion with uniform low echo located at the uncus of the pancreas.

Spiral CT (Somatom Sensation 16, Siemens, Munich, Germany) images were obtained prior to and at $30 \mathrm{~s}$ for the hepatic artery phase, $60 \mathrm{~s}$ for the portal venous phase, and $150 \mathrm{~s}$ for hepatic parenchymal phase after intravenous administration of contrast material (Omnipaque, GE health care, Fairfield, CT, USA; total of $80 \mathrm{ml}, 3 \mathrm{ml} / \mathrm{s}$, via cubital vein with a mechanical power injector). Spiral CT revealed a $2.2 \mathrm{~cm}$ round mass in the uncus of the pancreas, which was isodense compared with the pancreatic parenchyma, with the mean CT attenuation values being $46 \mathrm{HU}$ (Fig. 1). During hepatic artery phase and portal venous phase, the mass was hypodense compared with the enhanced pancreas, with the mean CT attenuation values being $56 \mathrm{HU}$ and $66 \mathrm{HU}$, respectively (Fig. 2). During the 


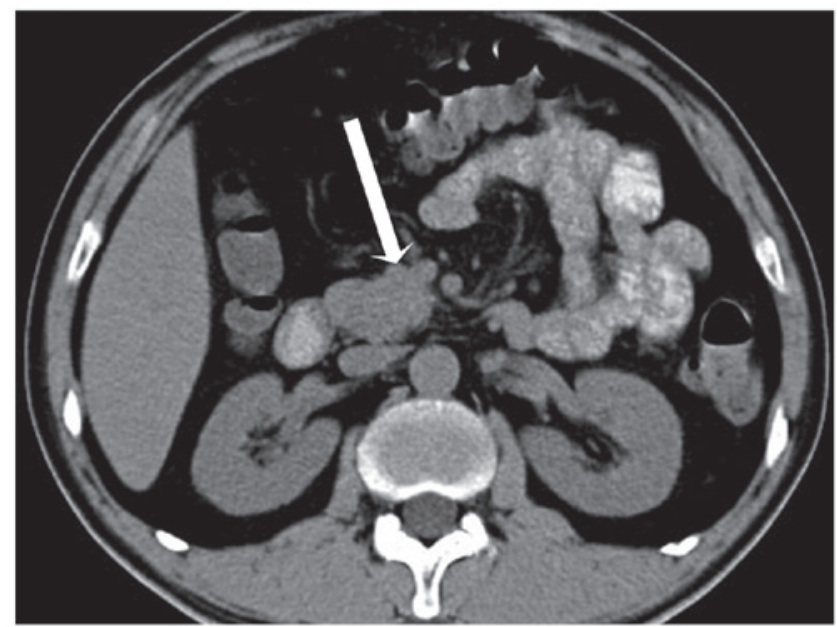

Figure 1. Pre-contrast CT of the abdomen illustrates a $2.2 \mathrm{~cm}$ round, isodense mass (white arrow) in the uncus of the pancreas, with the mean CT attenuation values of the tumor being $46 \mathrm{HU}$.

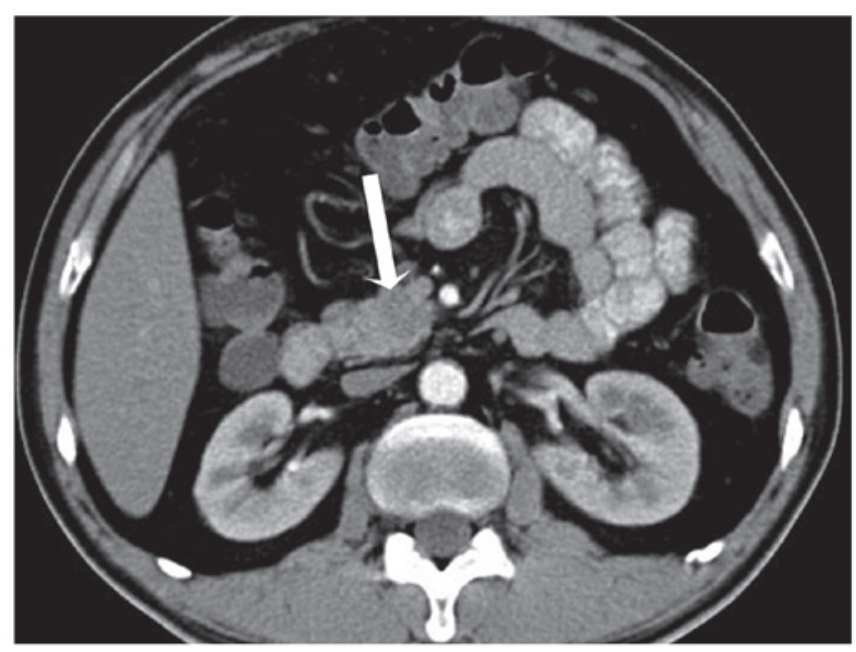

Figure 2. During the hepatic artery phase following bolus injection of intravenous contrast, CT demonstrates that the mass (white arrow) is hypodense compared with the enhanced pancreas, with the mean $\mathrm{CT}$ attenuation values of the tumor being $56 \mathrm{HU}$.

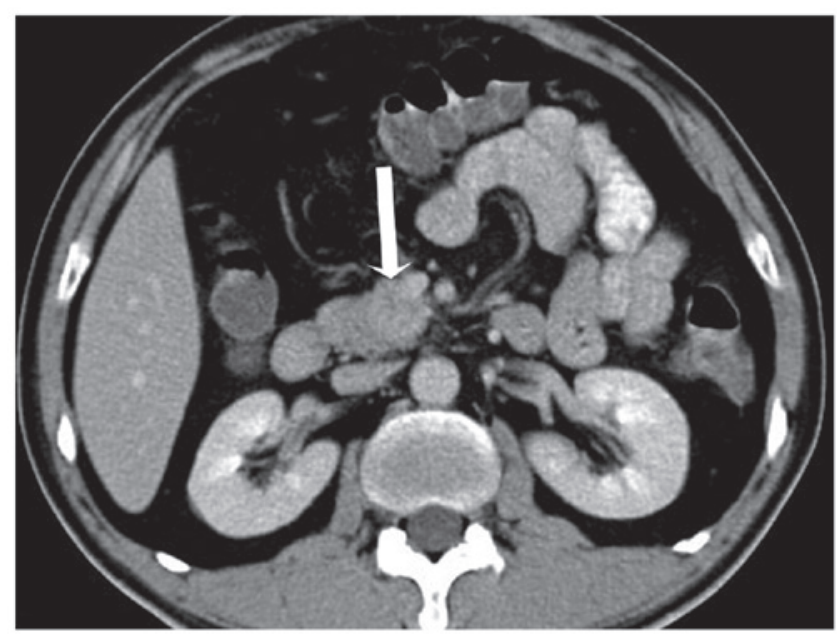

Figure 3. During the hepatic parenchymal phase, the mass (white arrow) became slightly hyperdense compared with the enhanced pancreas, with the mean $\mathrm{CT}$ attenuation values of the tumor being $74 \mathrm{HU}$.

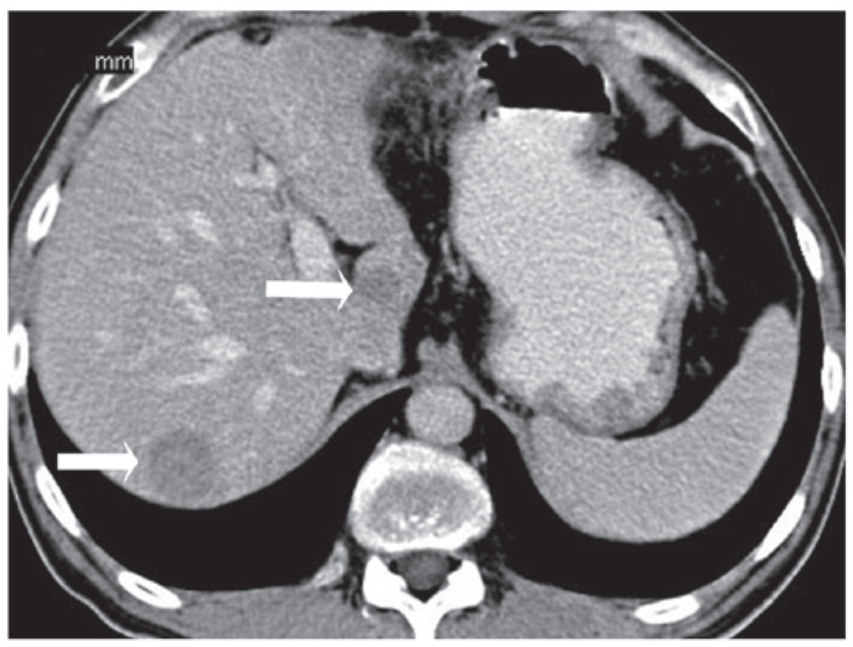

Figure 4. Two hypodense masses (white arrows) with minimal diffuse heterogeneous enhancement in the liver were observed in the patient.

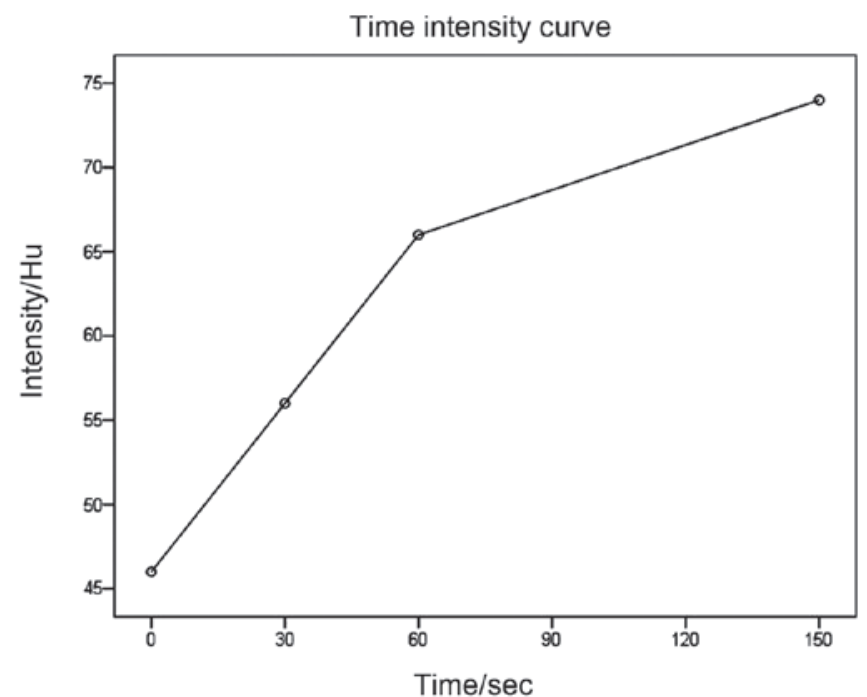

Figure 5. Time-intensity curve of the enhancement profile demonstrates a hypervascular, progressive-enhancement modality by the pancreatic VIPoma during the multiple-phase dynamic contrast-enhanced spiral CT scans.

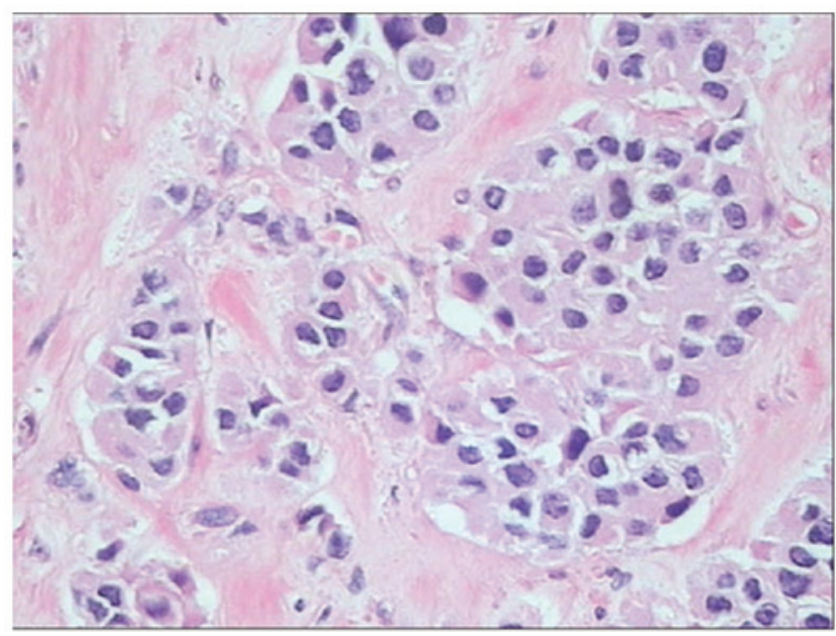

Figure 6. Histopathological analysis of the pancreatic mass reveals the classic neuroendocrine feature of nested cells, coexisting with marked fibrotic stroma (Hematoxylin and Eosin; magnification, x400). 
hepatic parenchymal phase, it became hyperdense with the mean CT attenuation values being $74 \mathrm{HU}$ (Fig. 3). The process of dynamic contrast-enhanced CT demonstrated a hypervascular progressive-strengthening modality (Fig. 4). There were multiple masses scattered throughout the liver that demonstrated hypodense lesions with minimal diffuse heterogeneous enhancement (Fig. 5).

Surgical exploration revealed a small, encapsulated, solid mass of $2.5 \mathrm{~cm}$ in size in the uncus of the pancreas. Pancreaticoduodenectomy and wedge resection of a number of liver metastases was undertaken. Histopathology of the pancreatic mass revealed the typical neuroendocrine features of nested cells, coexisting with marked fibrotic stroma (Fig. 6). The immunohistochemical analysis of the tumor for plasma VIP-immunoreactivity was markedly positive (+++), and the tumor was also stained slightly positive for other peptides, including NSE (++), AAT (+), AACT (++), CK-P (+), CgA (++), Syn (+), and negative for ACTH and insulin. These findings were consistent with the diagnosis criteria of VIPoma.

Postoperatively, the patient recovered immediately from the symptoms and there has been no evidence of recurrence during the past 27 months of follow-up since being discharged from the hospital on May 12, 2013.

\section{Discussion}

VIPomas are rare, hormone-producing tumors; the majority of cases (90\%) originate from endocrine pancreatic cells (6). The annual incidence of VIPoma is low and reported to be $\sim 1$ per 10 million people in the USA. VIPomas are more frequently diagnosed in women $(65 \%)$ compared with men $(35 \%)$, with the age of onset ranging between 2-83 years (mean age, 53.1 years). At the time of presentation, $\geq 70 \%$ of patients already have metastases identified, and the great majority of these tumors are malignant based on the presence of hepatic or lymph node involvement and other distant metastasis (1). In humans, the majority of VIPomas occur within the substance of the pancreas. Approximately $75 \%$ of VIPomas are localized in the body and tail of the pancreas, while the remaining $25 \%$ occur in the head of the pancreas (2). Clinically, production of large amounts of VIP hormone results in watery diarrhea, hypokalemia, and achlorhydria (7). This condition is called WDHA syndrome and was first described by Verner and Morrison in 1958 (7). The case described in the present study occurred in the uncus of the pancreas, and presented with WDHA syndrome, which was comparable with the literature. The fasting plasma VIP levels were $>200 \mathrm{pg} / \mathrm{ml}$ (normal, 0-190 pg/ml) are required to establish the diagnosis (2). The VIP-immunoreactivity of the present case was markedly positive $(+++)$, and combined with the WDHA syndrome, the diagnosis of VIPoma was confirmed.

The classic and most common enhancement CT pattern of syndromic pancreatic islet cell tumors illustrates a hyperattenuating lesion in the arterial phase that becomes inconspicuous in the venous phase $(5,8)$. However, to the best of our knowledge, only a few CT findings of pancreatic VIPoma have previously been reported (3-5) and the radiological manifestations of MPSCT have not previously been described. The previous studies demonstrated that VIPoma appears as a round, well-defined, homogeneous mass with central necrosis and hypervascularized; heterogeneous on contrast enhanced CT with internal septa, the CT attenuation values range between $23.4-46 \mathrm{HU}$ in the non-enhancement phase, and 76-116 HU following enhancement. In addition, a previous study illustrated that half of VIPomas present with calcification (5). In the present case, the lesion was isodense compared with the pancreatic parenchyma, and the process of contrast-enhanced multiple-phase CT demonstrated a progressive-strengthening modality, which was different from the previous comparable studies and not consistent with the classic CT appearances of syndromic pancreatic islet cell tumors.

VIPomas are hypervascularized and rich in tumor cells and fibrosis. Since fibrous stroma is less vascularized, this may result in contrast agent pooling within the tissue, and therefore the fibrous area may demonstrate relative hypoattenuation on early-phase images but hyperattenuation on late-phase images. Conversely, as the viable tumor cells area requires an increased blood supply, the development of a tumor is accompanied with hypervascularity, it demonstrate relative hyperattenuation on early-phase images (9). The present authors hypothesized that the progressive-strengthening modality of the VIPoma was mainly attributed to the large amount of fibrotic stroma, and its hypervascularization.

Another notable observation in the current patient was that, although the primary tumor measured only $2.2 \mathrm{~cm}$ in diameter, multiple metastatic tumors were present. Semelka et al (7) proposed that it may be a feature of VIPoma to present as a small primary pancreatic tumor in the setting of liver metastases. Comparably, other case studies have reported large tumors without liver metastases $(3,4)$. Therefore, we propose that a small, primary, pancreatic VIPoma may present with liver metastases while a large one may present without liver metastases.

There are numerous differential diagnoses that require consideration when a pancreatic mass is detected without evidence of increased hormone release. VIPoma primarily needs to be differentiated from pancreatic carcinoma, which is one of the most common space occupying lesions of the pancreas. Previous studies have demonstrated that contrast-enhancement images of pancreatic carcinoma during the early phase on spiral CT usually present as low attenuating lesions in comparison with the surrounding pancreatic parenchyma; while on the late phase imaging, the contrast on the pancreatic parenchyma generally reduces, resulting in carcinomas presenting as a progressive-strengthening modality as in the present case $(9,10)$. However, Hata et al $(9)$ performed dynamic CT studies in 20 patients with pancreatic carcinoma and demonstrated that the CT values (mean \pm SD) of pancreatic carcinomas after contrast injection were $28.3 \pm 12.8 \mathrm{HU}$ in the hepatic artery phase, $36.7 \pm 14.5 \mathrm{HU}$ in the hepatic portal venous phase, and $42.3 \pm 14.6 \mathrm{HU}$ in the hepatic parenchymal phase, respectively, which were notably lower compared with what was observed in the present case (56 HU, $66 \mathrm{HU}$ and $74 \mathrm{HU}$ in corresponding phase). In addition, pancreatic or bile duct dilatation or local extension or distant metastases may indicate pancreatic carcinoma. Although insulinoma, gastrioma, glucagonoma, somatostatinoma and nonfunctioning pancreatic endocrine 
tumors are all pancreatic endocrine tumors in addition to VIPoma, the majority of these tumors are hypervascular in nature, presenting as a hyperattenuating lesion in the arterial phase and becoming inconspicuous in the venous phase $(3,4)$, which is different to the present case. In addition, other pancreatic diseases, including mass-forming chronic pancreatitis, pancreatic metastases, tuberculosis, solid pseudopapillary tumor and lymphoma, should be considered in the further differential diagnosis. If combined with the history and clinical manifestations, the differentiation may be much easier.

At present, there is no consensus with regard to standard guidelines for the treatment of VIPoma, due to its rare occurrence, particularly in the uncus of the pancreas with liver metastases. Surgery appears to be the most effective means of treatment, and concurrent treatment with octreotide has advanced the preoperative electrolyte management (11). In addition, the combination of octreotide, chemotherapy, resection of tumor, radiofrequency tissue ablation and liver transplantation may be selected for metastatic VIPoma in the liver (11-13).

In summary, the present study reports a case of a small VIPoma, only $2.2 \mathrm{~cm}$ in diameter, arising from the region of the uncus of the pancreas with liver metastases. If a patient presents to hospital with the following: WDHA syndrome (watery diarrhea, hypokalemia, achlorhydria), particularly with watery diarrhea; markedly elevated VIP serum levels; hypervascular lesion; progressive-strengthening lesion; and with calcification in the pancreas, the diagnosis of the VIPoma should be considered and a small primary pancreatic tumor with liver metastases may also fit this diagnosis.

\section{References}

1. Ghaferi AA, Chojnacki KA, Long WD, Cameron JL and Yeo CJ: Pancreatic VIPomas: subject review and one institutional experience. J Gastrointest Surg 12: 382-393, 2008.

2. Delcore R and Friesen SR: Gastrointestinal neuroendocrine tumors. J Am Coll Surg 178: 187-211, 1994.

3. Tjon A, Tham RT, Jansen JB, et al: MR, CT, and ultrasound findings of metastatic Vipoma in pancreas. J Comput Assist Tomogr 13: 142-144, 1989.

4. Remme CA, de Groot GH and Schrijver G: Diagnosis and treatment of VIPoma in a female patient. Eur J Gastroenterol Hepatol 18: 93-99, 2006.

5. Horton KM, Hruban RH, Yeo C and Fishman EK. Multi-detector row CT of pancreatic islet cell tumors. Radiographics 26: 453-464, 2006.

6. Aspestrand F, Kolmannskog F and Jacobsen M. CT, MR imaging and angiography in pancreatic apudomas. Acta Radiol 34: 468-473, 1993.

7. Semelka RC, Custodio CM, Cem Balci N and Woosley JT. Neuroendocrine tumors of the pancreas: spectrum of appearances on MRI. J Magn Reson Imaging 11: 141-148, 2000.

8. Sheth S, Hruban RK and Fishman EK. Helical CT of islet cell tumors of the pancreas: typical and atypical manifestations. Am J Roentgenol 179: 725-730, 2002.

9. Hata H, Mori H, Matsumoto S, et al: Fibrous stroma and vascularity of pancreatic carcinoma: correlation with enhancement patterns on CT. Abdom Imaging 35: 172-180, 2010.

10. Lu DS, Vedantham S, Krasny RM, Kadell B, Berger WL and Reber HA: Two-phase helical CT for pancreatic tumors: pancreatic versus hepatic phase enhancement of tumor, pancreas, and vascular structures. Radiology 199: 697-701, 1996.

11. Song S, Shi R, Li B and Liu Y: Diagnosis and treatment of pancreatic vasoactive intestinal peptide endocrine tumors. Pancreas 38: 811-814, 2009.

12. Xiang G, Liu X, Tan C, Zhang H, Mai G and Zheng Z: Diagnosis and treatment of VIPoma: a case report and literature review in China. Pancreas 41: 806-807, 2012.

13. Peng SY, Li JT, Liu YB, et al: Diagnosis and treatment of VIPoma in China: (case report and 31 cases review) diagnosis and treatment of VIPoma. Pancreas 28: 93-97, 2004. 